\title{
Comprehending particle production at RHIC and LHC energies using global measurements
}

\author{
Sadhana Dash, ${ }^{1}$ Basanta K. Nandi, ${ }^{1}$ Ranjit Nayak, ${ }^{1}$ Ashutosh Kumar Pandey, ${ }^{1}$ and Priyanka Sett ${ }^{1}$ \\ ${ }^{1}$ Indian Institute of Technology Bombay, Mumbai, Indi *
}

(Dated: October 10, 2018)

\begin{abstract}
The centrality dependence of the charged-particle multiplicity densities $\left(d N_{c h} / d \eta\right)$ and transverse energy densities $\left(d E_{T} / d \eta\right)$ are investigated using the two-component Glauber approach for broad range of energies in heavy ion collisions at RHIC and LHC. A comprehensive study shows that the data is well described within the framework of two component model which includes the contribution of "soft processes" and "hard processes" for different centrality classes and energies. The data at two different energies are compared by means of the ratio of $d N_{c h} / d \eta$ (and $d E_{T} / d \eta$ ) to see the interplay of energy and $x$-scaling.
\end{abstract}

\section{INTRODUCTION}

Heavy ion collisions at relativistic energies provide means to study strongly-interacting matter are very high densities and temperatures. The charged particle multiplicity distribution has been one of the most widely studied experimental observable to comprehend the hot and dense matter created in such collisions and in general to understand the particle production mechanism in such systems. Another important observable which gives similar insight is the distribution of the transverse energy, $E_{T}$ in such collisions. Experimentally, the aforementioned global distributions are quantified by the charged particle multiplicity density $\left(d N_{c h} / d \eta\right)$ and the transverse energy density $\left(d E_{T} / d \eta\right)$. In heavy ion collisions, "hard" scattering processes, characterized by the production of high transverse momentum $\left(p_{T}\right)$ particles, are expected to scale with the number of collisions, $N_{\text {coll }}$ while, the production of low $p_{T}$ particles (from soft processes) is expected to scale with the number of participants, $N_{\text {part }}[1]$. The dependence of the $d N_{c h} / d \eta$ and $d E_{T} / d \eta$ with collision energy and centrality gives important information on the relative contribution of hard and soft processes to the particle production mechanism. In this paper, we have used three approaches to study the variation of $d N_{c h} / d \eta$ (and $d E_{T} / d \eta$ ) with centrality and beam energy. The first approach is inspired by the two-component models which decompose nucleus-nucleus collisions into soft and hard interactions, where the soft interactions produce particles with an average multiplicity proportional to $N_{\text {part }}$ and the probability for hard interactions to occur is proportional to $N_{\text {coll }}$ [1]. The two component approach [1] parametrizes $d N_{c h} / d \eta$ by the following expression.

$$
\frac{d N_{c h}}{d \eta}=n_{p p}\left((1-x) \frac{\left\langle N_{\text {part }}\right\rangle}{2}+x\left\langle N_{\text {coll }}\right\rangle\right)
$$

In this model, $n_{p p}$ represents the average charged-particle multiplicity in a single nucleon-nucleon collision, while $x$ refers to the fraction of "hard" partonic interactions. In a previous study [2], the variation of $d N_{c h} / d \eta$ was described by using the above model where both $n_{p p}$ and $x$ were treated as free parameters. As $n_{p p}$ represents a physical observable (mean multiplicity in $p p$ collisions), the above approach might not give the proper values and dependence of $x$ and $n_{p p}$ with collision energy and centrality. The obtained values for the fit parameters might be an artifact of the fitting procedure. The method can be improvised by constraining the parameter $n_{p p}$ by using the experimentally measured values at a given energy. This would result in a robust extraction of $x$ parameter with respect to centrality and beam energy. In other two approaches, $d N_{c h} / d \eta$ was studied by single component parameterization [3 6]. A similar approach was also used to explain the variation of $d E_{T} / d \eta$ dependence with collision centrality and energy. In the case of $d E_{T} / d \eta$, we do not have a broad experimental measurement of $E_{T_{p p}}$ (for nucleon nucleon collisions) and hence mean $E_{T_{p p}}$ was treated as a free parameter. We have used the $x$ parameter values obtained from the $d N_{c h} / d \eta$ fit. This assumption seems valid as the $x$ value would remain same for similar centralities and energies. This method can provide an estimation of variation of $E_{T_{p p}}$ with collision energy.

\section{ANALYSIS}

The data for $d N_{c h} / d \eta$ and $d E_{T} / d \eta$ for $\mathrm{Au}+\mathrm{Au}$ collisions at mid-rapidity have been presented by PHENIX experiment for a large set of energies at RHIC [7]. The LHC data for $d N_{c h} / d \eta$ in $\mathrm{Pb}+\mathrm{Pb}$ collisions at $2.76 \mathrm{TeV}$ and 5.02 TeV has been taken from ALICE experiment [6, 8] and the $d E_{T} / d \eta$ data for $2.76 \mathrm{TeV}$ can be found in Ref. 9]. As discussed in previous section, the values of $n_{p p}$ were taken to be the experimentally measured values for a particular energy. Since we do not have experimental measurement of charged-particle multiplicity

\footnotetext{
* sadhana@phy.iitb.ac.in
} 


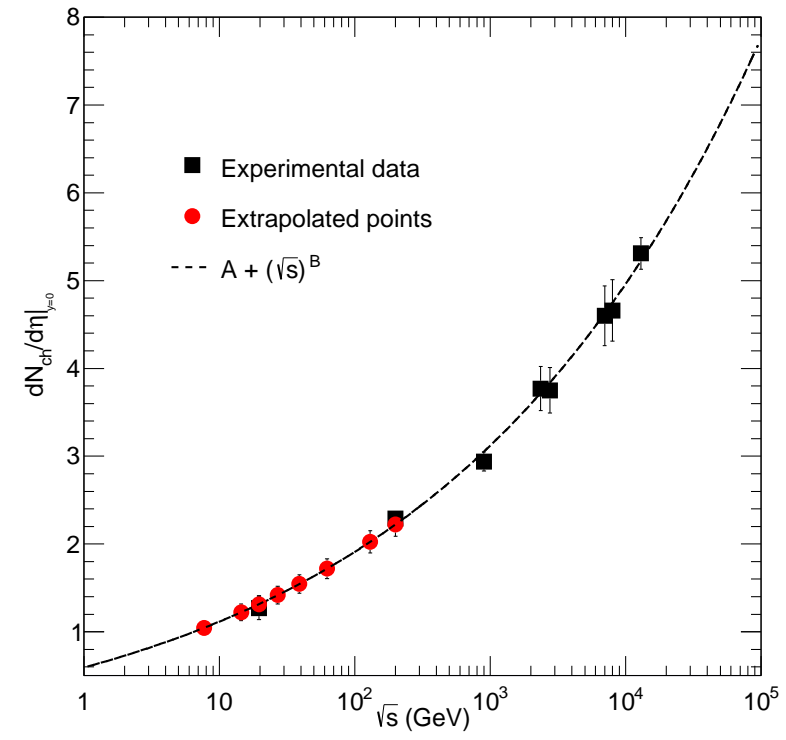

FIG. 1. The charged particle multiplicity density measured in $p p$ collisions at RHIC [11] and LHC [12, 13] energies. The solid squares are the experimentally measured $n_{p p}$ data. The data is described by a power law of the form $A+\sqrt{s}^{B}$, shown by the dashed line. The solid circles are the extrapolated data for the RHIC beam scan energies.

in $p p$ collisions for most of the beam energy scan energies at RHIC, the values for $n_{p p}$, were obtained by extrapolating the $\sqrt{s}$ dependence of measured $n_{p p}$ in $p p$ collisions at different energies. This is depicted in Figure 1 The measured experimental data from RHIC [11] and LHC [12, 13] are shown by the solid squares. The data is described by a power law shown by the dashed line. The closed circles are the estimated $n_{p p}$ values for the beam scan energies at RHIC. To compare the two-component model with the measured data, one also needs the values of $\left\langle N_{\text {part }}\right\rangle$ and $\left\langle N_{\text {coll }}\right\rangle$. The values of $\left\langle N_{\text {part }}\right\rangle$ are obtained from the Monte-Carlo Glauber model [10] which simulates the heavy ion collision process on an event by event basis. The values of $\left\langle N_{\text {coll }}\right\rangle$ can be obtained by a simple power law parameterization of $\left\langle N_{\text {part }}\right\rangle$ obtained from the Monte-Carlo Glauber model [10]. The dependence is given by, $N_{\text {coll }}=a \times N_{\text {part }}{ }^{b}$. The dependence of the parameters for various collision energies is shown in Table I. The value of the parameter $x$ is obtained from the fit to the data employing Eq. 1.

The $d N_{c h} / d \eta$ has also been described by single parameter of the following forms $[3]$, $]$,

$$
\begin{aligned}
& d N_{c h} / d \eta \propto\left\langle N_{\text {part }}\right\rangle^{\alpha} \\
& d N_{c h} / d \eta \propto\left\langle N_{\text {coll }}\right\rangle^{\beta}
\end{aligned}
$$

The analysis of $d E_{T} / d \eta$ was also carried in a similar manner with a slight modification. In this case, the values

\begin{tabular}{|c|c|c|}
\hline \hline$\sqrt{s_{N N}}$ & $a$ & $b$ \\
\hline $7.7 \mathrm{GeV}$ & $0.315 \pm 0.099$ & $1.34 \pm 0.057$ \\
$19.6 \mathrm{GeV}$ & $0.312 \pm 0.096$ & $1.35 \pm 0.055$ \\
$27 \mathrm{GeV}$ & $0.306 \pm 0.1$ & $1.36 \pm 0.058$ \\
$39 \mathrm{GeV}$ & $0.301 \pm 0.096$ & $1.36 \pm 0.056$ \\
$62.4 \mathrm{GeV}$ & $0.395 \pm 0.097$ & $1.32 \pm 0.054$ \\
$130 \mathrm{GeV}$ & $0.367 \pm 0.144$ & $1.35 \pm 0.073$ \\
$200 \mathrm{GeV}$ & $0.356 \pm 0.117$ & $1.36 \pm 0.065$ \\
$2.76 \mathrm{TeV}$ & $0.365 \pm 0.052$ & $1.41 \pm 0.025$ \\
$5.02 \mathrm{TeV}$ & $0.352 \pm 0.523$ & $1.42 \pm 0.027$ \\
\hline \hline
\end{tabular}

TABLE I. The parameters $a$ and $b$ obtained from the parameterization of $N_{\text {part }}$ and $N_{\text {coll }}$ for different collision energies.

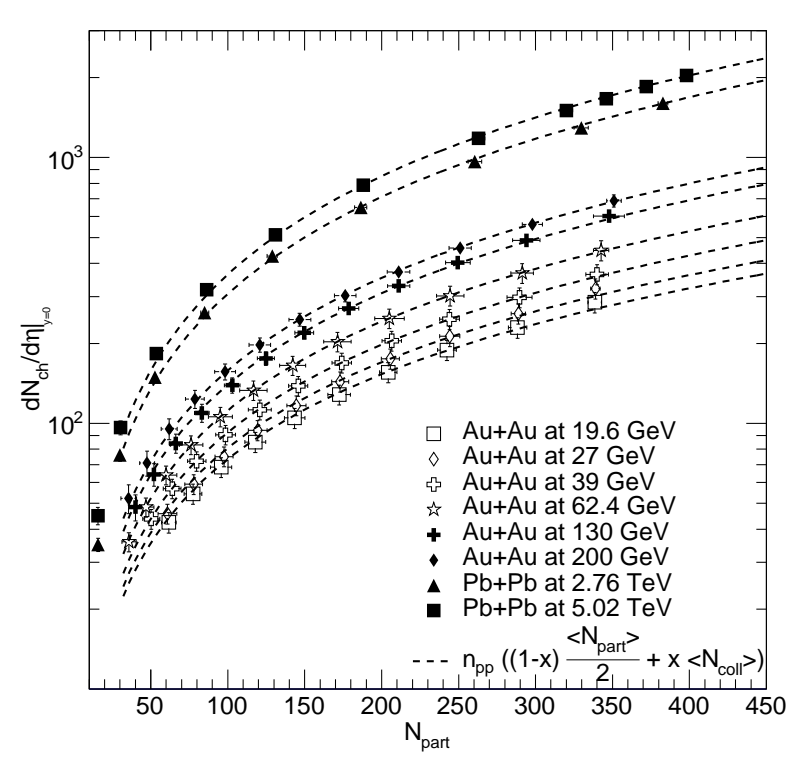

FIG. 2. Charged particle multiplicity densities measured in heavy ion collisions at $\operatorname{RHIC}(\mathrm{Au}+\mathrm{Au})$ [7] and LHC $(\mathrm{Pb}+\mathrm{Pb})$ [6, 8] energies. The dashed line represents the fits to Eq. 1 .

of $x$ parameter obtained from the $d N_{c h} / d \eta$ (Eq. 1) fits were used while the the mean transverse energy obtained in $p p$ collision was treated as a free parameter. This is done as we do not have published experimental results of mean transverse energy in $p p$ collisions at different energies. The above approach would also give us the energy dependence of the mean transverse energy in $p p$ collisions $\left(E_{T_{p p}}\right)$. In addition, the pairwise ratios between the $d N_{c h} / d \eta$ and $d E_{T} / d \eta$ per participant pairs for two different energy sets were also evaluated. The ratios were calculated by interpolating the values of charged particle densities at a particular energy to the same $N_{\text {part }}$ values at a different energy. 


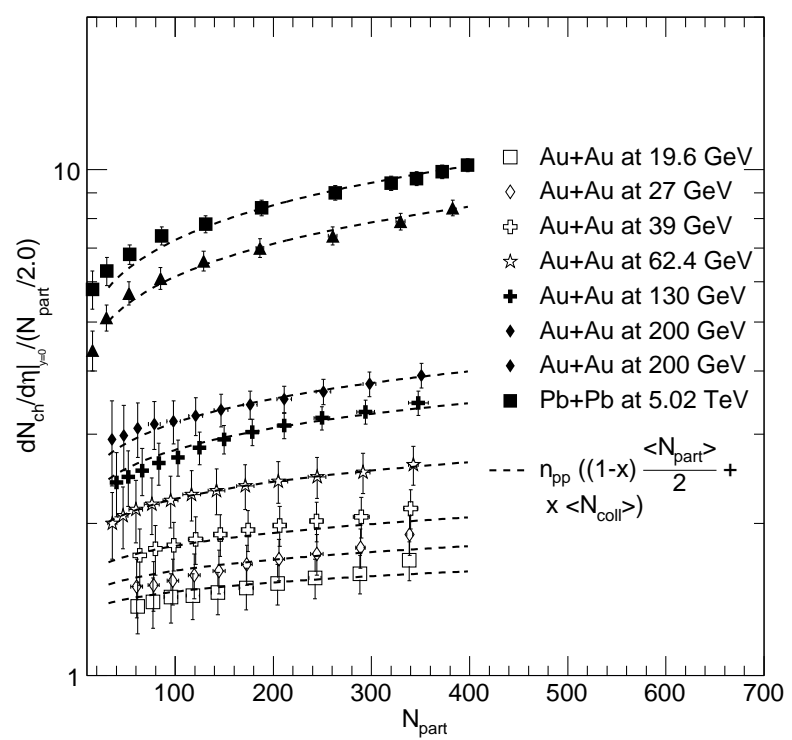

FIG. 3. Charged particle multiplicity densities scaled by the number of participants measured in heavy ion collisions at RHIC $(\mathrm{Au}+\mathrm{Au})$ [7] and $\mathrm{LHC}(\mathrm{Pb}+\mathrm{Pb})$ [6, 8] energies. The dashed line represents the fits to Eq. 1

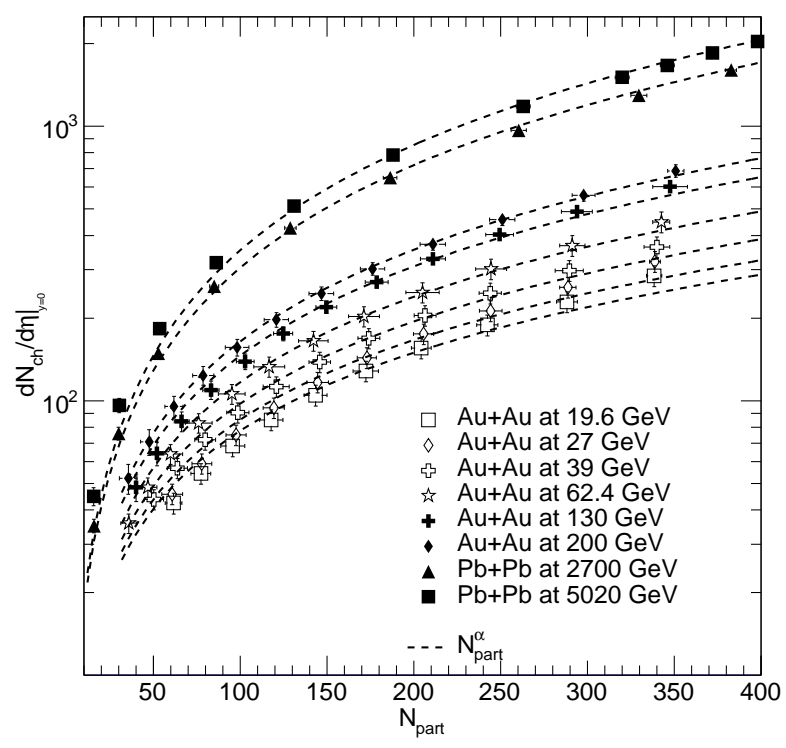

FIG. 4. Charged particle multiplicity densities measured in heavy ion collisions at RHIC $(\mathrm{Au}+\mathrm{Au})$ [7] and LHC $(\mathrm{Pb}+\mathrm{Pb})$ 6, 8] energies. The dashed line represents the fits to Eq. 2

\section{RESULTS : CHARGED PARTICLE MULTIPLICITY DENSITIES}

The results obtained from the two component (Eq. 1) fits of $d N_{c h} / d \eta$ measured at RHIC ([7]) and LHC $([6,[8])$ energies are shown in Figure 2] The dashed lines show the two-component fits performed by $\chi^{2}$ minimization.

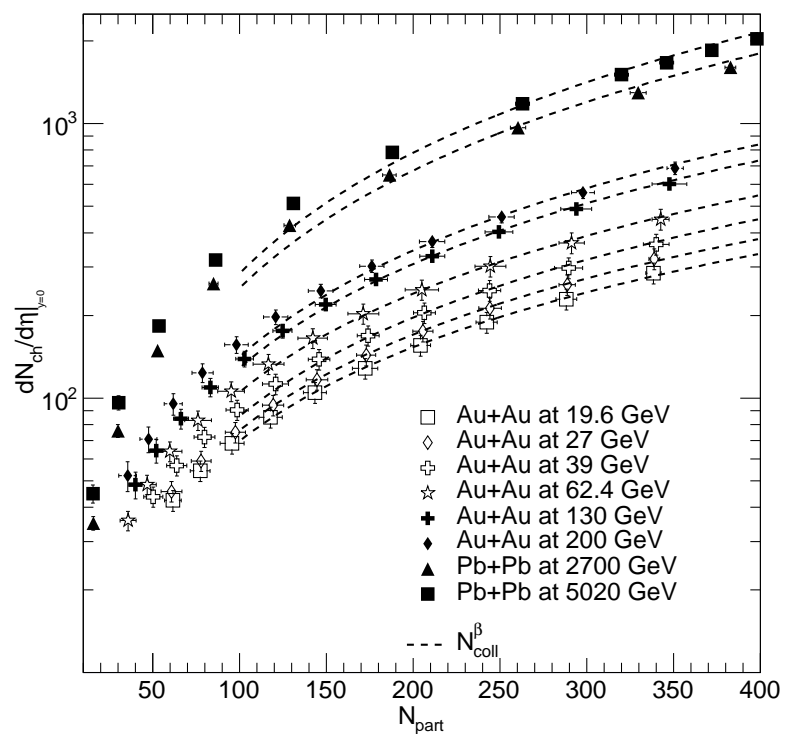

FIG. 5. Charged particle multiplicity densities measured in heavy ion collisions at $\operatorname{RHIC}(\mathrm{Au}+\mathrm{Au})$ [7] and $\mathrm{LHC}$ $(\mathrm{Pb}+\mathrm{Pb})$ [6, [8] energies. The dashed line represents the fits to $\mathrm{Eq} .3$

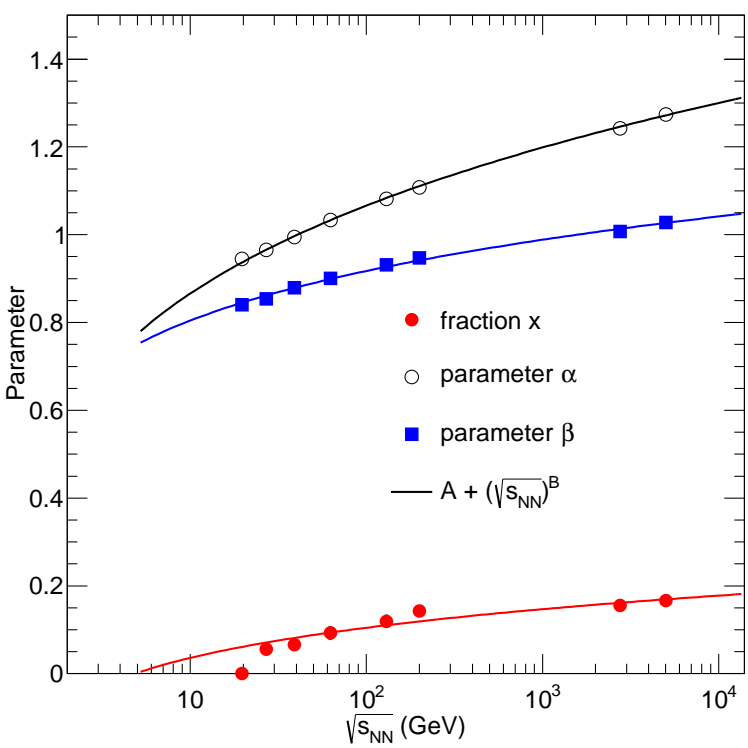

FIG. 6. The variation of the parameters $x$ (solid circles), $\alpha$ (open circles) and $\beta$ (solid squares) as a function of $\sqrt{s_{N N}}$. The solid lines are the power law parameterization.

Figure 3 shows the same data but the multiplicity densities are scaled with the number of participant pairs. One can observe from Figure 3, that at RHIC energies, the scaled multiplicity densities do not have a strong dependence on the $\left\langle N_{\text {part }}\right\rangle$ and have a flat behavior. However, the observed trend for LHC differs from RHIC . As a function of system size, the scaled multiplicity density shows an increasing trend, which one can expect from 


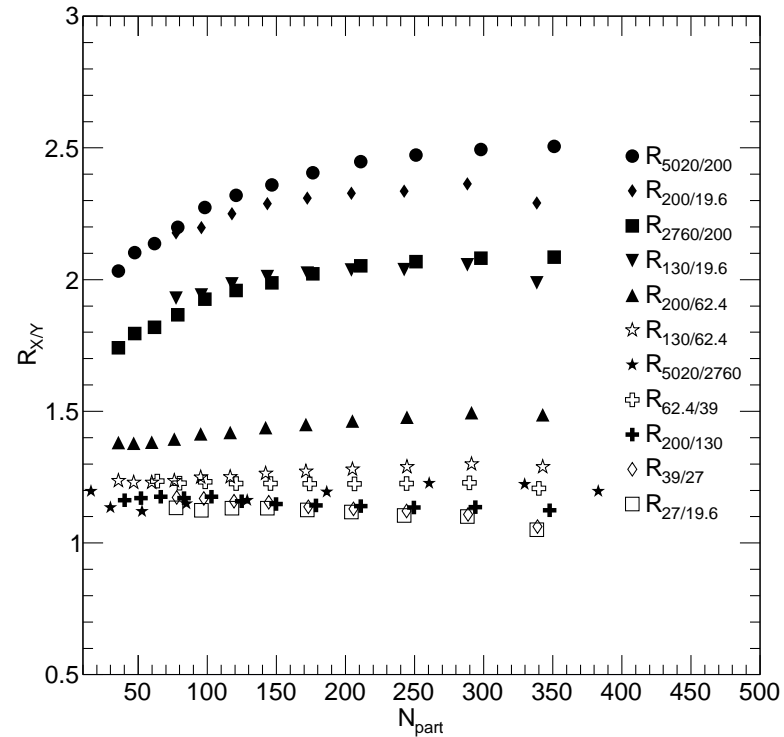

FIG. 7. The variation of the double ratio of the charged particle multiplicity density (refer text) as a function of $\left\langle N_{\text {part }}\right\rangle$.

\begin{tabular}{|c|c|c|c|}
\hline \hline$\sqrt{s_{N N}}$ & $x$ & $\alpha$ & $\beta$ \\
\hline $19.6 \mathrm{GeV}$ & $0.056 \pm 0.013$ & $0.95 \pm 0.006$ & $0.84 \pm 0.006$ \\
$27 \mathrm{GeV}$ & $0.066 \pm 0.013$ & $0.97 \pm 0.006$ & $0.85 \pm 0.006$ \\
$39 \mathrm{GeV}$ & $0.093 \pm 0.014$ & $0.99 \pm 0.006$ & $0.88 \pm 0.006$ \\
$62.4 \mathrm{GeV}$ & $0.12 \pm 0.014$ & $1.03 \pm 0.006$ & $0.90 \pm 0.006$ \\
$130 \mathrm{GeV}$ & $0.14 \pm 0.009$ & $1.08 \pm 0.006$ & $0.93 \pm 0.004$ \\
$200 \mathrm{GeV}$ & $0.16 \pm 0.009$ & $1.11 \pm 0.004$ & $0.94 \pm 0.004$ \\
$2.76 \mathrm{TeV}$ & $0.17 \pm 0.006$ & $1.24 \pm 0.003$ & $1.01 \pm 0.003$ \\
$5.02 \mathrm{TeV}$ & $0.18 \pm 0.004$ & $1.27 \pm 0.002$ & $1.02 \pm 0.002$ \\
\hline \hline
\end{tabular}

TABLE II. The parameters $x, \alpha$ and $\beta$ obtained from the fits of multiplicity density distributions using Eq. 1. Eq. 2 and Eq. 3 for different collision energies.

the large difference in collision energies.

The fits for the single component parameterizations, Eq. 2 and Eq. 3 are shown in Figure 4 and Figure 5 respectively. Figure 4 shows a good agreement of the parameterization with the measured data for all energies. While using the parameterization stated in Eq. 3, we restrict the fit in the common $\left\langle N_{\text {part }}\right\rangle$ region to have a consistent approach.

All three parameters, namely $x, \alpha$ and $\beta$ are also studied as a function of $\sqrt{s_{N N}}$ and are shown in Figure 6 . One can observe an increasing trend with energy. This is expected as the number of hard scattering processes increase with an increase in number of collisions which increases with energy. The increasing trend can be analytically described by a power law, $A+{\sqrt{s_{N N}}}^{B}$. Table凹 summarizes the value of the parameters extracted from the fit.

We also compare the pair wise ratios at two differ-

\begin{tabular}{|c|c|c|c|c|}
\hline \hline$X(\mathrm{GeV})$ & $Y(\mathrm{GeV})$ & $\begin{array}{c}R_{\sqrt{s_{N N}}} \\
=X / Y\end{array}$ & $\begin{array}{c}R_{x} \\
=X_{x} / Y_{x}\end{array}$ & $R_{\sqrt{s_{N N}}} \times R_{x}$ \\
\hline 5020 & 200 & 25.1 & 1.13 & 28.39 \\
200 & 19.6 & 10.2 & 2.77 & 28.27 \\
130 & 19 & 6.63 & 2.55 & 16.9 \\
2760 & 200 & 13.8 & 1.07 & 14.8 \\
200 & 62 & 3.21 & 1.3 & 4.18 \\
130 & 62 & 2.08 & 1.2 & 2.49 \\
62 & 39 & 1.6 & 1.29 & 2.06 \\
39 & 27 & 1.44 & 1.41 & 2.03 \\
5020 & 2760 & 1.82 & 1.06 & 1.92 \\
200 & 130 & 1.54 & 1.09 & 1.67 \\
27 & 19 & 1.378 & 1.17 & 1.61 \\
\hline \hline
\end{tabular}

TABLE III. The ratio of $X / Y$ and the ratio of the $x$ fraction of the corresponding center of mass energies.

ent set of energies as shown in Figure 7 A variable $R_{X / Y}=\left.\frac{d N / d \eta}{\left\langle N_{\text {part }}\right\rangle / 2}\right|_{X} /\left.\frac{d N / d \eta}{\left\langle N_{\text {part }}\right\rangle / 2}\right|_{Y}$, is used to denote the magnitude of the ratio of multiplicity densities at energy $X$ and energy $Y$ (where $X>Y$ ). The ratio is evaluated for similar $\left\langle N_{\text {part }}\right\rangle$ values for both the energies considered [2]. The ratio shows a flat behavior as a function of system size for collision energy ranges which differs by a factor of 3 or less i.e. $R_{27 / 19.6}, R_{39 / 27}, R_{62.4 / 39.0}$ and $R_{200 / 130}$. However, as the value of the collision energy differs by a factor of 4 or more, the magnitude of the ratio increases, as well as, the ratio shows an increasing trend as a function of $\left\langle N_{\text {part }}\right\rangle$. There is an increase of $\sim 20 \%$ from most peripheral to most central collisions. On careful observation, one can see that the trend does not follow strictly the energy difference only. For example, the values of $R_{2760 / 200}$ is consistently lower than $R_{200 / 19.6}$ although the collision energy difference is more for the former than the later. Therefore, one should also take into account an increase in the $x$ factor along with an increase of energy. The interplay of both these factors i.e the energy difference and the increased contribution of the hard scattering component determines the observed trend. The contribution from both the factors are tabulated in [II].

\section{RESULTS : TRANSVERSE ENERGY DENSITY}

The measured transverse energy density, $d E_{T} / d \eta$ as a function of $\left\langle N_{\text {part }}\right\rangle$ is studied by the two-component model [1] approach described in previous sections. As discussed in the method for $d E_{T} / d \eta$, the $x$ parameter values were used from the fits of centrality dependence of $d N_{c h} / d \eta$ while $E_{T_{p p}}$ was treated as a free parameter. The results of the fit are shown in Figure 8, The model describes the data very nicely for all energies from RHIC 


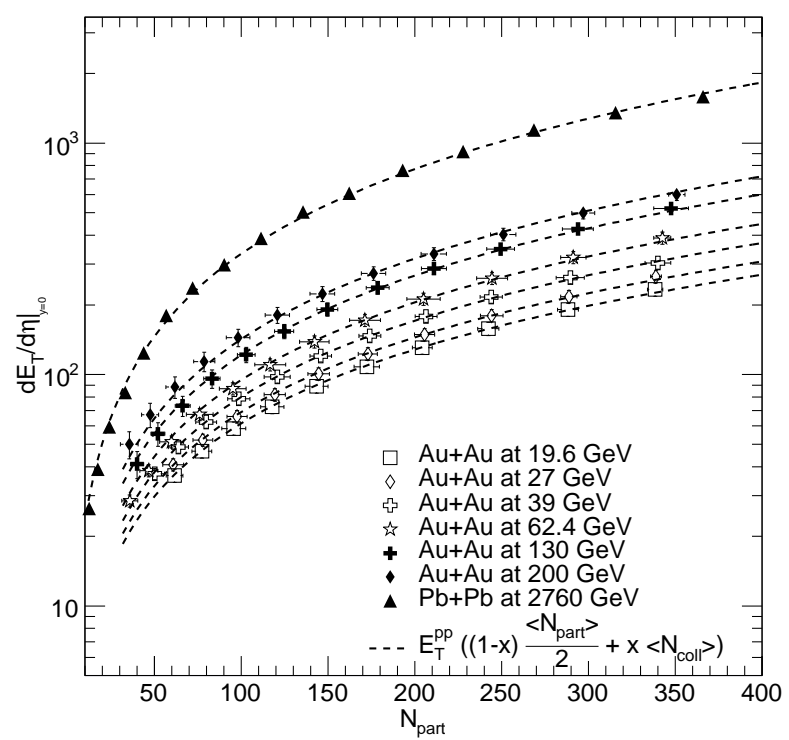

FIG. 8. Charged particle transverse energy densities measured in heavy ion collisions at RHIC $(\mathrm{Au}+\mathrm{Au})$ [7] and $\mathrm{LHC}$ $(\mathrm{Pb}+\mathrm{Pb})$ [9] energies. The dashed line represents the fits to Eq. 1$]$

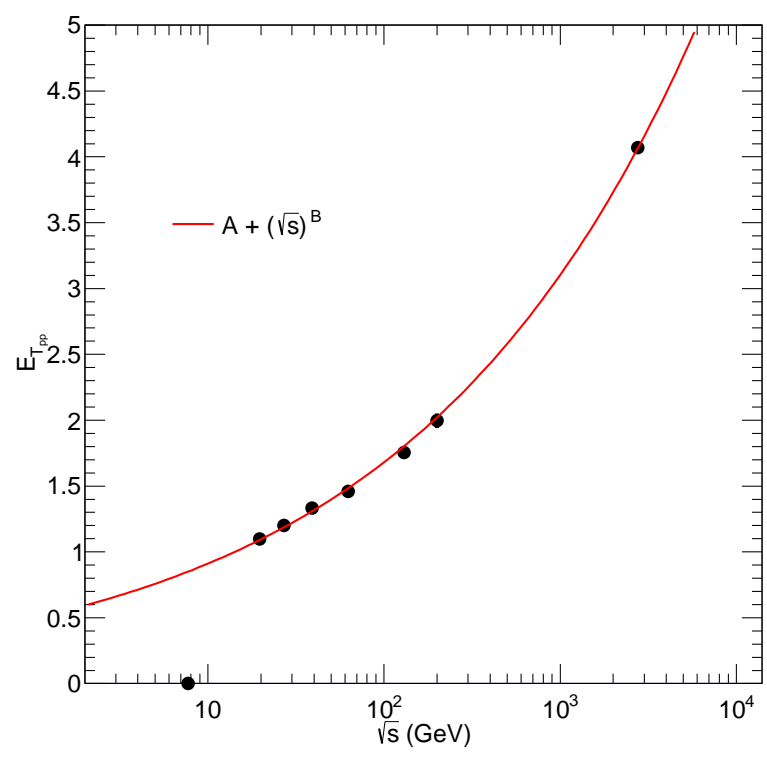

FIG. 9. The variation of $E_{T_{p p}}$ as a function of center of mass energy. This is parameterized by a power law shown by the solid line.

to LHC. The extracted values of $E_{T_{p p}}$ is plotted as a function of $\sqrt{s}$ in Figure 9. The values are parameterized with the same function as that of $n_{p p}$ as depicted in Figure 9, Figure 10 depicts the scaled transverse energy densities as a function of centrality for various energies. The scaled energy densities do not have any centrality dependence. This behaviour is also seen in the pair wise ratios of $d E_{T} / d \eta$ at two different set of energies as shown in

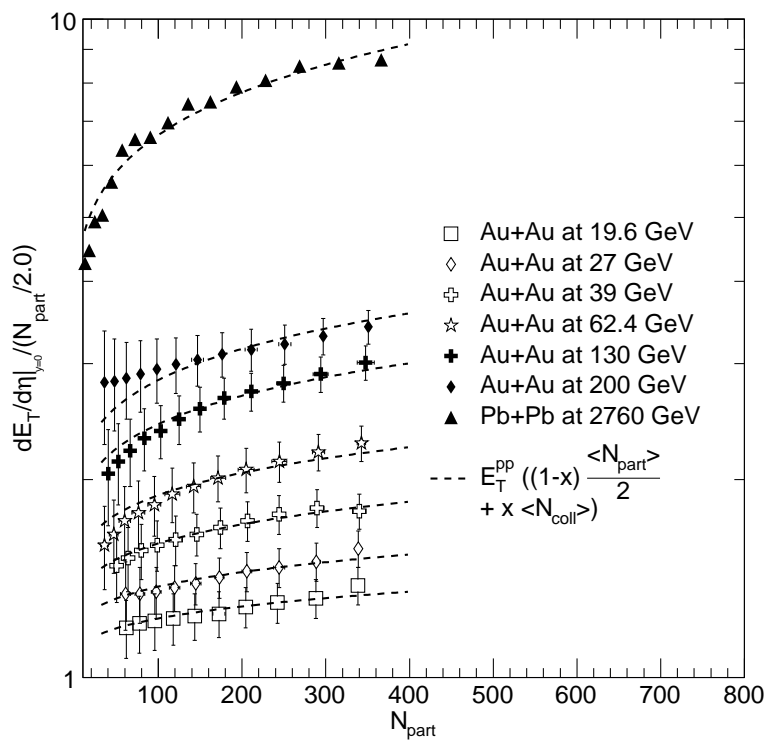

FIG. 10. Charged particle transverse energy densities scaled by the number of participants measured in heavy ion collisions at RHIC $(\mathrm{Au}+\mathrm{Au})$ [7] and $\mathrm{LHC}(\mathrm{Pb}+\mathrm{Pb})$ [9] energies. The dashed line represents the fits to Eq. 1.

Figure 11. A variable $R_{X / Y}^{E_{T}}=\left.\frac{d E_{T} / d \eta}{\left\langle N_{\text {part }}\right\rangle / 2}\right|_{X} /\left.\frac{d E_{T} / d \eta}{\left\langle N_{\text {part }}\right\rangle / 2}\right|_{Y}$, is used to denote the magnitude of the ratio of transverse energy densities at energy $X$ and energy $Y$. The ratio shows a flat behavior as a function of system size for all the collision energy ranges considered. The magnitude of the ratios depends on the factor of difference in energy. For example, the values of $R_{2760 / 200}$ is comparable to that of $R_{200 / 19.6}$ as the collision energy difference is more or less of same order. The same was not observed for case of the multiplicity densities. The values of $R_{2760 / 200}$ is consistently higher than $R_{130 / 19.6}$ inspite of the $x$ factor being higher in the later. This was not seen in the trend of pairwise ratios of multiplicity densities where both the ratios were comparable. One can conclude that the role of $x$ is not significant in dictating the observed trend in case of transverse energy densities.

\section{SUMMARY}

The midrapidity charged-particle multiplicity density and the transverse energy density measurements were described nicely by the two-component Glauber approach for a broad range of energies. The analysis shows that the fractional contribution of "hard processes" parametrized by the value of $x$ increases with an increase in beam energy. A centrality dependence of the same is also shown but the increase is not very significant with respect to centrality. The pair wise ratios of the multiplicity densities at two different energies shows a scaling which depends on the interplay of energy difference and the contribution from the hard scattering component. The later 


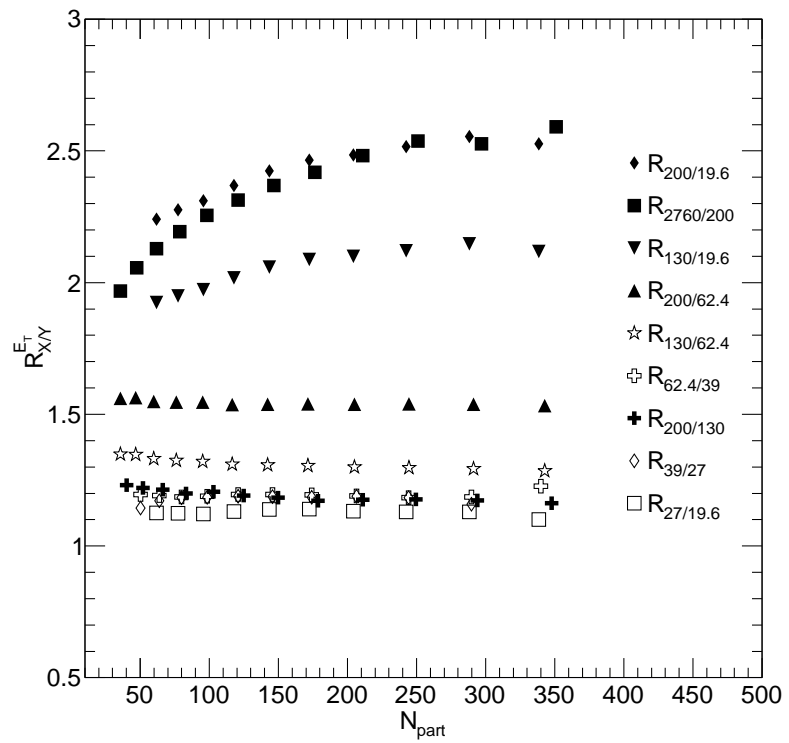

FIG. 11. The variation of the double ratio of the charged particle energy density (refer text) as a function of $\left\langle N_{\text {part }}\right\rangle$. plays no role in the observed order of ratios for the transverse energy densities. Although the model is bit crude and simplistic in its approach, the description of the the overall features of the centrality dependence of multiplicity and transverse energy densities at various energies is in good agreement with the data.
[1] D. Kharzeev and M. Nardi, Phys. Lett. B507, 121 (2001).

[2] L. Zhou and G. S.F. Stephans, Phys. Rev. C90, 014902 (2014).

[3] M. C. Abreu et al., (NA50 Collaboration), Phys. Rev. Lett. B530, 43 (2002).

[4] M. M. Aggarwal et al., (WA98 Collaboration), Eur. Phys. J. C18, 651 (2001).

[5] F. Antinori et al., (NA57 and WA97 Collaboration), J. Phys. G27, 391 (2001).

[6] K. Aamodt et al., (ALICE Collaboration), Phys. Rev. Lett. 106, 032301 (2011).

[7] A. Adare et al., (PHENIX Collaboration), Phys. Rev.
C93, 024901 (2016).

[8] J. Adam et al., (ALICE Collaboration), Phys. Rev. Lett. 116, no.22, 222302 (2016).

[9] C. Loizides for ALICE Collaboration, J. Phys. G38, 124040 (2011).

[10] M. M. Miller et al., Ann. Rev. Nucl. Part. Sci. 57, 205243 (2007).

[11] B. B. Back et al., (PHOBOS Collaboration), Phys. Rev. C70, 021902 (2004).

[12] J. Adam et al., (ALICE Collaboration), arXiv:1509.07541 nucl-ex].

[13] J. Adam et al., (ALICE Collaboration), Phys. Lett. B753, 319-329 (2016). 


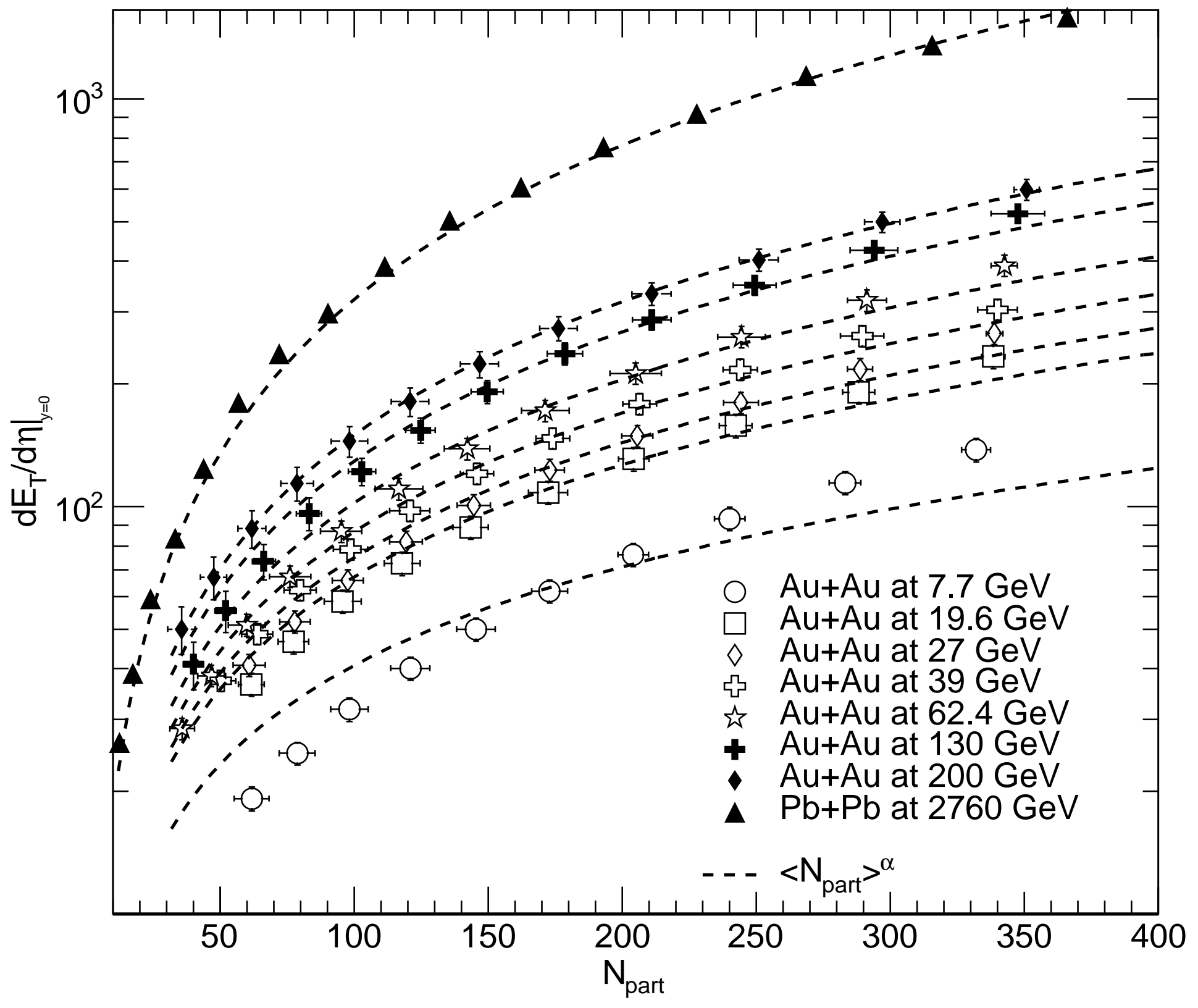




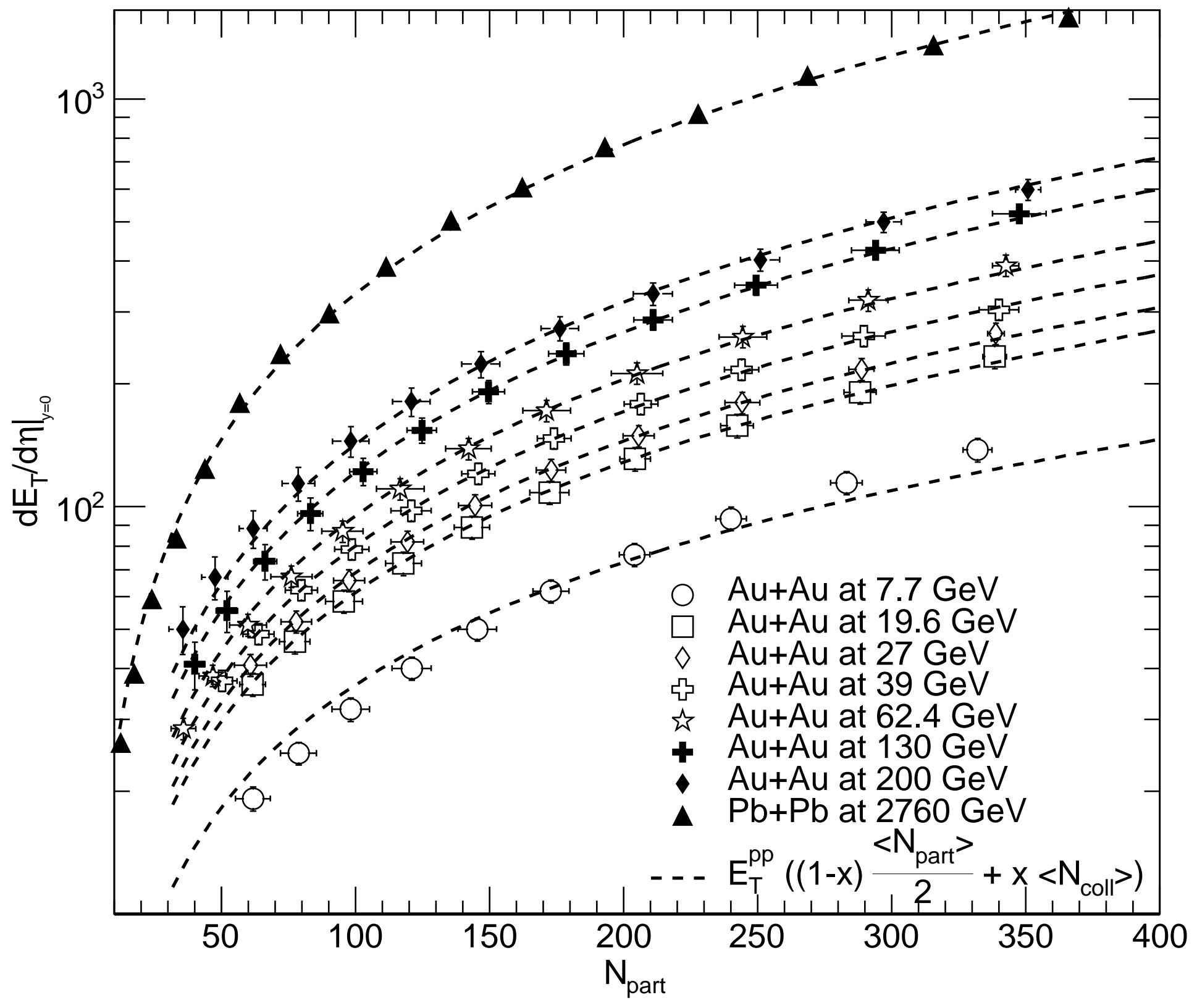


